\title{
A Novel Synthesis of Flavones from 2-Methoxybenzoic Acids
}

\author{
Jae In Lee, Hwa Soo Son. and Mi Gung Jung \\ Department of Chemistry, College of Natural Science, Duksung Women's University, Seoul 132-714, Korea \\ "E-mail:jilee@duksung.ac.kr \\ Received July 29, 2005
}

Key Words : Flavones, 2'-Methoxyacetophenones, 1-(2-Methoxyphenyl)-3-phenyl-1,3-propanediones, Condensation

The flavones (2-phenylchromones) are widely distributed in vascular plants ${ }^{1}$ and have attracted a lot of attention because they possess biological activities, such as antioxidant effect, antiviral activity, and anticarcinogenic effect. ${ }^{2}$ The main synthetic methods known for the flavones are the cyclodehydration of 1-(2-hydroxyphenyl)-3-phenyl-1,3propanediones, the oxidative cyclization of 2'-hydroxychalcones, and synthesis via an intramolecular Wittig reaction. $^{3}$ The rearrangement of benzoyl esters of $2^{2}$ hydroxyacetophenones (Baker-Venkataraman process ${ }^{4}$ ) and the direct benzoylation of 2'-hydroxyacetophenones with benzoyl chlorides" or methyl benzoates ${ }^{6}$ affords 1-(2hydroxyphenyl)-3-phenyl-1,3-propanediones, which are cyclodehydrated to give flavones in acidic conditions. The treatment of 2'-hydroxychalcones which are prepared from 2 -hydroxyacetophenones and benzaldehydes in the presence of 2 equiv of lithium disopropylamide with oxidizing agents also affords flavones at high temperature. ${ }^{7}$ Alternatively Wittig reaction ${ }^{8}$ involves the intramolecular olefination of phosphoranes obtained from triphenylphosphine and 2-acetoxyphenacyl bromides, a four step process from 2'-hydroxyacetophenones. A common feature in all these methods is that they invariably use 2'hydroxyacetophenones as the starting material.

However, there are no reports of the synthesis of flavones from 2 -methoxyacetophenones. Although 1-(2-methoxyphenyl)-3-methyl-1,3-propanedione is cyclized with boiling
HI to give 2-methylchromone, the scope of the reaction is not fully investigated and there are no reports on the synthesis of flavones with 2-substituted phenyl group. ${ }^{9}$ Furthermore, it has been reported that the condensation of 2'-methoxyacetophenone with methyl 2-methoxybenzoate using sodium or sodium hydride failed to produce the corresponding 1,3-diketone. ${ }^{10}$

As part of our continuing studies of flavonoids, ${ }^{11}$ we report that flavones can be newly synthesized in two steps via 1-(2-methoxyphenyl)-3-phenyl-1,3-propanediones from 2'-methoxyacetophenones cheaper than 2'-hydroxyacetophenones in general. 2'-Methoxyacetophenones 2 were readily prepared by the treatment of 2-methoxybenzoic acids 1 with 2 equiv of methyllithium in THF for $2 \mathrm{~h}$ at $-78^{\circ} \mathrm{C}$ (Scheme 1). The reaction proceeded smoothly to give 2 free from the corresponding tertiary alcohols after acidic hydrolysis $\left(\mathrm{R}^{1}, \mathrm{R}^{2}, \mathrm{R}^{3}=\mathrm{H} ; 92 \%, \mathrm{R}^{1}=\mathrm{OCH}_{3}, \mathrm{R}^{2}, \mathrm{R}^{3}=\mathrm{H} ; 93 \%\right.$, $\mathrm{R}^{\prime}, \mathrm{R}^{3}=\mathrm{H}, \mathrm{R}^{2}=\mathrm{OCH}_{3} ; 88 \%$ ). However, the reaction of $2,6-$ dimethoxybenzoic acid with methyllithium proceeded sluggish due to the steric effect and thus 2',6'-dimethoxyacetophenone was prepared by the treatment of $N$-methoxy$N$-methyl 2,6-dimethoxybenzamide with methyl magnesium bromide at room temperature in $75 \%$ yield.

The key intermediates, 1-(2-methoxyphenyl)-3-phenyl1,3-propanediones $\mathbf{4}$, for the synthesis of flavones 5 were readily prepared by the condensation of the lithium enolates of 2 with benzoyl cyanides. To find out the optimum reagent<smiles>[R]c1cc(OC)c(C(=O)O)c([R])c1[R]</smiles>

1<smiles>[R]c1cc(OC)c(C(C)=O)c([R])c1[R]</smiles>

2

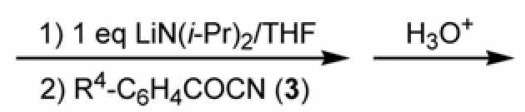

2) $\mathrm{R}^{4}-\mathrm{C}_{6} \mathrm{H}_{4} \operatorname{COCN}(3)$<smiles>[R4]c1ccc(-c2cc(=O)c3c([R])c([R])c([R])cc3o2)cc1</smiles>

5

$\mathrm{R}^{1}, \mathrm{R}^{2}, \mathrm{R}^{3}=\mathrm{H}, \mathrm{OCH}_{3}(\mathrm{OH}) ; \mathrm{R}^{4}=\mathrm{H}, \mathrm{OCH}_{3}, \mathrm{Cl}$ 
for the benzoylation of 2, benzoyl chloride, 2-pyridyl benzoate, and benzoyl cyanide were added to the lithium enolate solution in $\mathrm{THF}$ at $-78^{\circ} \mathrm{C}$, which was generated from 2'-methoxyacetophenone and 1 equiv of lithium diisopropylamide for $2 \mathrm{~h}$ at $-20^{\circ} \mathrm{C}$. The resulting yellow solution was allowed to warm to room temperature and 1-(2methoxyphenyl)-3-phenyl-1,3-propanedione was obtained in $63 \%, 51 \%, 94 \%$ yield, respectively, after chromatographic separation. The condensation of the lithium enolate of 2 with 3 worked well regardless of the kind of substituents (methoxy, chloro) on both 2 '-methoxyacetophenones and benzoyl cyanides under the present reaction conditions and 4 were obtained in high yields ( $80-95 \%)$. The 'H NMR spectra of $\mathbf{4}$ showed the presence of enolic OH ( $\delta$ $16.00-16.20)$ together with the vinyl protons $(\delta 7.09-7.20)$ and also indicated that enols are major tautomers in all of products.

The cyclization of 4 was successfully accomplished by heating with hydriodic acid in glacial acetic acid. The initial cyclization of 1-(2-methoxyphenyl)-3-phenyl-1,3-propanedione with sulfuric acid, hydrobromic acid, and hydriodic acid in acetonitrile didn't proceed at room temperature. However, the cyclization accompanied by the cleavage of the 2-methoxy group of 1-(2-methoxyphenyl)-3-phenyl-1,3propanedione with $47 \% \mathrm{HI}$ proceeded well in glacial acetic acid for $1.5 \mathrm{~h}$ at $100^{\circ} \mathrm{C}$ to afford flavone in $78 \%$ yield. The use of $48 \% \mathrm{HBr}$ was also effective, but the yield of flavone was decreased to $55 \%$.

As shown in Table 1, various flavones were synthesized in overall high yields (47-67\%) from the starting 2-methoxybenzoic acids. The present method was generally applicable for the synthesis of 5 having methoxy and chloro substituents on the A- and/or B-ring. Thus, the reaction worked well both for the methoxy substituent ( $5 \mathbf{d}-5 \mathrm{~g}$ ) on the A-ring and the methoxy $(\mathbf{5 b}, \mathbf{5 e})$ or chloro substituent $(\mathbf{5 c}, \mathbf{5 f})$ on the B-ring of 5. During the cyclization 6 or 7 -methoxy group of A-ring and 4'-methoxy group of B-ring were not cleaved under the present reaction conditions. However, the treatment of 1-(2,6-dimethoxyphenyl)-3-(4'-chlorophenyl)1,3-propanedione with $47 \% \mathrm{HI}$ in glacial acetic acid at reflux resulted in the cleavage of the two methoxy groups and the successive cyclization to produce 5-hydroxy-4'chloroflavone ( 5 h) in $85 \%$ yield.

Table 1. Preparation of Flavones from 2-Methoxybenzoic Acids

\begin{tabular}{cccccc}
\hline $\begin{array}{c}\text { Entry } \\
\mathbf{5}\end{array}$ & $\mathrm{R}^{\mathrm{l}}$ & $\mathrm{R}^{2}$ & $\mathrm{R}^{j}$ & $\mathrm{R}^{4}$ & $\begin{array}{c}\text { Isolated } \\
\text { yield, } \%\end{array}$ \\
\hline $\mathbf{a}$ & $\mathrm{H}$ & $\mathrm{H}$ & $\mathrm{H}$ & $\mathrm{H}$ & 67 \\
$\mathbf{b}$ & $\mathrm{H}$ & $\mathrm{H}$ & $\mathrm{H}$ & $\mathrm{OMe}$ & 55 \\
$\mathbf{c}$ & $\mathrm{H}$ & $\mathrm{H}$ & $\mathrm{H}$ & $\mathrm{Cl}$ & 63 \\
$\mathbf{d}$ & $\mathrm{OMe}$ & $\mathrm{H}$ & $\mathrm{H}$ & $\mathrm{H}$ & 66 \\
$\mathbf{e}$ & $\mathrm{OMe}$ & $\mathrm{H}$ & $\mathrm{H}$ & $\mathrm{OMe}$ & 47 \\
$\mathbf{f}$ & $\mathrm{OMe}$ & $\mathrm{H}$ & $\mathrm{H}$ & $\mathrm{Cl}$ & 53 \\
$\mathbf{g}$ & $\mathrm{H}$ & $\mathrm{OMe}$ & $\mathrm{H}$ & $\mathrm{H}$ & 54 \\
$\mathbf{h}$ & $\mathrm{H}$ & $\mathrm{H}$ & $\mathrm{OH}$ & $\mathrm{Cl}$ & 51 \\
\hline
\end{tabular}

"Overall yields of three steps from the starting 2-methoxybenzoic acids.
In conclusion, the present method provides some advantages over previous methods using 2'-hydroxyacetophenone derivatives with respect to (i) the cheapness of 2 -methoxyacetophenone derivatives in general (ii) the use of 1 equiv of lithium diisopropylamide for the benzoylation of $\mathbf{2}$ (iii) the high yield synthesis of $\mathbf{4}$ using benzoyl cyanides.

\section{Experimental Section}

Preparation of $2^{\prime}, 4^{\prime}$-dimethoxyacetophenone (General procedure). To a solution of 2,4-dimethoxybenzoic acid $(819.8 \mathrm{mg}, 4.5 \mathrm{mmol})$ in THF $(18 \mathrm{~mL})$ was slowly added methyllithium (1.5 M in $\mathrm{Et}_{2} \mathrm{O}, 6.0 \mathrm{~mL}, 9.0 \mathrm{mmol}$ ) under argon atmosphere at $-78^{\circ} \mathrm{C}$. After being stirred for $1 \mathrm{~h}$, the mixture was quenched with $0.5 \mathrm{~N}-\mathrm{HCl}(3 \mathrm{~mL})$ and $\mathrm{THF}$ was evaporated in vacto. The mixture was poured into $0.5 \mathrm{~N}$ $\mathrm{HCl}(30 \mathrm{~mL})$, extracted with methylene chloride $(3 \times 25$ $\mathrm{mL}$ ), and washed with sat. $\mathrm{NaHCO}_{3}(30 \mathrm{~mL})$. The combined organic phases were dried over $\mathrm{MgSO}_{4}$, filtered, and concentrated in vacuo. The residue was purified by vacuum distillation using Kugelrohr apparatus to give 2',4'-dimethoxyacetophenone $(754.1 \mathrm{mg}, 93 \%)$. ${ }^{1} \mathrm{H}$ NMR $(300 \mathrm{MHz}$, $\left.\mathrm{CDCl}_{3}\right) \delta 7.81(\mathrm{~d}, J=8.7 \mathrm{~Hz}, 1 \mathrm{H}), 6.49\left(\mathrm{dd}, J_{1}=8.7 \mathrm{~Hz}, J_{2}=\right.$ $2.3 \mathrm{~Hz}, 1 \mathrm{H}), 6.43(\mathrm{~d}, J=2.3 \mathrm{~Hz}, 1 \mathrm{H}), 3.86(\mathrm{~s}, 3 \mathrm{H}), 3.82(\mathrm{~s}$, 3H), 2.56 (s, 3H); FT-IR (film) 3004, 2943, 1661 (C=O), $1598,1465,1358,1269,1027,827,734 \mathrm{~cm}^{-1}$; Ms $m / z(\%)$ $180\left(\mathrm{M}^{+}, 61\right), 166(29), 165(100), 135(14), 107(22), 92$ (15), 77 (18).

Preparation of 1-(2,4-dimethoxyphenyl)-3-phenyl-1,3propanedione (General procedure). To a solution of $2^{\prime}, 4^{\prime}-$ dimethoxyacetophenone $(630.7 \mathrm{mg}, 3.5 \mathrm{mmol})$ in THF (12 $\mathrm{mL}$ ) was added lithium diisopropylamide $(2.0 \mathrm{M}, 1.8 \mathrm{~mL}$, $3.6 \mathrm{mmol}$ ) under argon atmosphere at $-20^{\circ} \mathrm{C}$. The stirring was continued for $2 \mathrm{~h}$ at this temperature and a solution of benzoyl cyanide $(459.0 \mathrm{mg}, 3.5 \mathrm{mmol})$ in THF $(6 \mathrm{~mL})$ was added at $-78^{\circ} \mathrm{C}$. After being stirred for $2 \mathrm{~h}$ between $-78^{\circ} \mathrm{C}$ and room temperature, the mixture was quenched with 0.5 $\mathrm{N}-\mathrm{HCl}(3 \mathrm{~mL})$, and THF was evaporated in vacuo. The mixture was poured into $0.5 \mathrm{~N}-\mathrm{HCl}(30 \mathrm{~mL})$, extracted with methylene chloride $(3 \times 25 \mathrm{~mL})$, and washed with brine $(30$ $\mathrm{mL}$ ). The combined organic phases were dried over $\mathrm{MgSO}_{4}$, filtered, and concentrated in vacuo. The residue was purified by silica gel column chromatography using 30\% EtOAc/ $n$-hexane to give 1-(2,4-dimethoxyphenyl)-3-phenyl-1,3propanedione $(915.5 \mathrm{mg}, 92 \%)$. M.p. $37-40^{\circ} \mathrm{C}$; 'H NMR $\left(300 \mathrm{MHz}, \mathrm{CDCl}_{3}\right)$ enolic form $\delta 16.20(\mathrm{~s}, 1 \mathrm{H}), 8.01(\mathrm{~d}, J=$ $8.4 \mathrm{~Hz}, 1 \mathrm{H}), 7.94-7.97(\mathrm{~m}, 2 \mathrm{H}), 7.47-7.50(\mathrm{~m}, 3 \mathrm{H}), 7.18(\mathrm{~s}$, $1 \mathrm{H}), 6.60\left(\mathrm{dd}, J_{1}=8.4 \mathrm{~Hz}, J_{2}=2.4 \mathrm{~Hz}, 1 \mathrm{H}\right), 6.52(\mathrm{~d}, J=2.4$ $\mathrm{Hz}, 1 \mathrm{H}), 3.95(\mathrm{~s}, 3 \mathrm{H}), 3.88(\mathrm{~s}, 3 \mathrm{H})$; FT-IR (KBr) 3061, 3004, $2967,1607,1504,1277,1026,775 \mathrm{~cm}^{-1}$; Ms $m / z(\%) 284$ $\left(\mathrm{M}^{+}, 20\right), 253(81), 207(49), 165(100), 138(25), 105(16)$, $77(20)$.

Preparation of 7-methoxyflavone $5 \mathrm{~d}$ (General procedure). A solution of 1-(2,4-dimethoxyphenyl)-3-phenyl-1,3propanedione $(852.9 \mathrm{mg}, 3.0 \mathrm{mmol})$ and hydriodic acid $(47$ wt. $\%$ in $\mathrm{H}_{2} \mathrm{O}, 1.09 \mathrm{~mL}, 6.0 \mathrm{mmol}$ ) in glacial acetic acid (12 $\mathrm{mL}$ ) was refluxed for $1.5 \mathrm{~h}$ at $100^{\circ} \mathrm{C}$. After evaporation of 
acetic acid, the mixture was poured into sat. $\mathrm{NaHCO}_{3}(30$ $\mathrm{mL}$ ), and the aqueous phase was extracted with methylene chloride $(3 \times 20 \mathrm{~mL})$. The combined organic phases were dried over $\mathrm{MgSO}_{4}$, filtered, and concentrated in vacuo. The crude product was purified by silica gel column chromatography using $40 \% \mathrm{EtOAc} / n$-hexane to give $5 \mathrm{~d}(583.0 \mathrm{mg}$, $77 \%$ ). M.p. $109-111^{\circ} \mathrm{C}$ (lit. ${ }^{7 \mathrm{a}} 110-112^{\circ} \mathrm{C}$ ); ${ }^{1} \mathrm{H}$ NMR $(300$ $\left.\mathrm{MHz}, \mathrm{CDCl}_{3}\right) \delta 8.11(\mathrm{~d}, J=8.7 \mathrm{~Hz}, 1 \mathrm{H}), 7.87-7.90(\mathrm{~m}, 2 \mathrm{H})$, 7.48-7.52 (m, 3H), 6.95-6.98 (m, 2H), $6.74(\mathrm{~s}, 1 \mathrm{H}), 3.92(\mathrm{~s}$, $3 \mathrm{H})$; FT-IR ( $\mathrm{KBr}) 3065,2985,1641(\mathrm{C}=\mathrm{O}), 1439,1163,770$ $\mathrm{cm}^{-1}$; Ms $m / z(\%) 252\left(\mathrm{M}^{+}, 100\right), 224(42), 209(55), 150$ (22), $122(19)$.

Flavone (5a). M.p. $95-96{ }^{\circ} \mathrm{C}$ (lit. ${ }^{70} 97-98{ }^{\circ} \mathrm{C}$ ); 'H NMR $\left(300 \mathrm{MHz}, \mathrm{CDCl}_{3}\right) \delta 8.24\left(\mathrm{dd}, J_{1}=7.8 \mathrm{~Hz}, J_{2}=1.5 \mathrm{~Hz}, 1 \mathrm{H}\right.$ ), 7.91-7.95 (m, 2H), 7.68-7.73 (m, $1 \mathrm{H}), 7.52-7.59(\mathrm{~m}, 4 \mathrm{H})$, 7.41-7.45 (m, 1H), $6.83(\mathrm{~s}, 1 \mathrm{H})$; FT-IR (KBr) 3054, 1644 $(\mathrm{C}=\mathrm{O}), 1422,1129,769 \mathrm{~cm}^{-1}$; Ms $m / z(\%) 222\left(\mathrm{M}^{+}, 100\right)$, $221(36), 194(46), 120(48), 92(30)$.

4'-Methoxyflavone (5b). M.p. $158-159^{\circ} \mathrm{C}$ (lit." $158-160$ $\left.{ }^{\circ} \mathrm{C}\right) ;{ }^{1} \mathrm{H}$ NMR $\left(300 \mathrm{MHz}, \mathrm{CDCl}_{3}\right) \delta 8.23\left(\mathrm{dd}, J_{1}=7.8 \mathrm{~Hz}, J_{2}\right.$ $=1.5 \mathrm{~Hz}, 1 \mathrm{H}), 7.89(\mathrm{~d}, J=9.0 \mathrm{~Hz}, 2 \mathrm{H}), 7.66-7.72(\mathrm{~m}, 1 \mathrm{H})$, $7.57(\mathrm{~d}, J=8.4 \mathrm{~Hz}, 1 \mathrm{H}), 7.39-7.44(\mathrm{~m}, 1 \mathrm{H}), 7.03(\mathrm{~d}, J=9.0$ $\mathrm{Hz}, 2 \mathrm{H}), 6.76(\mathrm{~s}, 1 \mathrm{H}), 3.90(\mathrm{~s}, 3 \mathrm{H})$; FT- $\mathrm{R}(\mathrm{KBr}) 3050,2992$, $1641(\mathrm{C}=\mathrm{O}), 1607,1466,1376,837 \mathrm{~cm}^{-1}$; Ms $m / z(\%) 252$ $\left(\mathrm{M}^{+}, 100\right), 251(33), 207(31), 132(51)$.

4'-Chloroflavone (5c). M.p. 185-187 ${ }^{\circ} \mathrm{C}$ (lit. ${ }^{\text {th }} 185-188$ $\left.{ }^{\circ} \mathrm{C}\right) ;{ }^{1} \mathrm{H}$ NMR ( $\left.300 \mathrm{MHz}, \mathrm{CDCl}_{3}\right) \delta 8.24\left(\mathrm{dd}, J_{1}=8.0 \mathrm{~Hz}, J_{2}\right.$ $=1.5 \mathrm{~Hz}, 1 \mathrm{H}), 7.86(\mathrm{~d}, J=8.7 \mathrm{~Hz}, 2 \mathrm{H}), 7.69-7.74(\mathrm{~m}, 1 \mathrm{H})$, $7.56(\mathrm{~d}, J=8.4 \mathrm{~Hz}, 1 \mathrm{H}), 7.51(\mathrm{~d}, J=8.7 \mathrm{~Hz}, 2 \mathrm{H}), 7.41-7.45$ $(\mathrm{m}, 1 \mathrm{H}), 6.79(\mathrm{~s}, 1 \mathrm{H})$; FT-IR (KBr) 3090, $1645(\mathrm{C}=\mathrm{O}), 1606$, $1467,1095,834 \mathrm{~cm}^{-1}$; Ms m/z (\%) $258\left(\mathrm{M}^{+}+2,34\right), 256\left(\mathrm{M}^{+}\right.$, $100), 230(14), 228(41), 120(57), 92(33)$.

4',7-Dimethoxyflavone (5e). M.p. $143-144{ }^{\circ} \mathrm{C}$ (lit. ${ }^{7 a} 142-$ $\left.143^{\circ} \mathrm{C}\right)$; 'H NMR $\left(300 \mathrm{MHz}, \mathrm{CDCl}_{3}\right) \delta 8.13(\mathrm{~d}, J=9.0 \mathrm{~Hz}$, $1 \mathrm{H}), 7.86(\mathrm{~d}, J=9.0 \mathrm{~Hz}, 2 \mathrm{H}), 7.02(\mathrm{~d}, J=9.0 \mathrm{~Hz}, 2 \mathrm{H}), 6.95-$ $6.99(\mathrm{~m}, 2 \mathrm{H}), 6.69(\mathrm{~s}, 1 \mathrm{H}), 3.93(\mathrm{~s}, 3 \mathrm{H}), 3.89(\mathrm{~s}, 3 \mathrm{H})$; FT-IR (KBr) 3080, 2940, $1641(\mathrm{C}=\mathrm{O}), 1606,1422,1376,1163$ $\mathrm{cm}^{-1}$; Ms $m / 2(\%) 282\left(\mathrm{M}^{+}, 100\right), 281(35), 239(29), 132(35)$.

4'-Chloro-7-methoxyflavone (5f). M.p. $171-173^{\circ} \mathrm{C}$ (lit." $\left.172-174^{\circ} \mathrm{C}\right)$; 'H NMR $\left(300 \mathrm{MHz}, \mathrm{CDCl}_{3}\right) \delta 8.13(\mathrm{~d}, J=8.7$ $\mathrm{Hz}, 1 \mathrm{H}), 7.84(\mathrm{~d}, J=8.7 \mathrm{~Hz}, 2 \mathrm{H}), 7.49(\mathrm{~d}, J=8.7 \mathrm{~Hz}, 2 \mathrm{H})$, $6.99\left(\mathrm{dd}, J_{1}=8.7 \mathrm{~Hz}, J_{2}=2.1 \mathrm{~Hz}, 1 \mathrm{H}\right), 6.95(\mathrm{~d}, J=2.1 \mathrm{~Hz}$, $1 \mathrm{H}), 6.73(\mathrm{~s}, 1 \mathrm{H}), 3.94(\mathrm{~s}, 3 \mathrm{H})$; FT-IR $(\mathrm{KBr}) 2986,1656$ $(\mathrm{C}=\mathrm{O}), 1607,1439,1374,1163,837 \mathrm{~cm}^{-1}$; Ms $m / z(\%) 288$ $\left(\mathrm{M}^{+}+2,34\right), 286\left(\mathrm{M}^{+}, 100\right), 260(14), 258(42), 243(51), 207$ (31), $150(30)$.

6-Methoxyflavone (5g). M.p. 161-163 ${ }^{\circ} \mathrm{C}$; ' H NMR (300 $\left.\mathrm{MHz}, \mathrm{CDCl}_{3}\right) \delta 7.91-7.94(\mathrm{~m}, 2 \mathrm{H}), 7.50-7.61(\mathrm{~m}, 5 \mathrm{H}), 7.26-$ $7.32(\mathrm{~m}, 1 \mathrm{H}), 6.83(\mathrm{~s}, 1 \mathrm{H}), 3.92(\mathrm{~s}, 3 \mathrm{H}) ;{ }^{13} \mathrm{C}$ NMR $(75 \mathrm{MHz}$, $\left.\mathrm{CDCl}_{3}\right) \delta 178.7,163.5,157.4,151.4,132.2,131.9,129.4$, $126.6,124.9,124.2,119.9,107.2,105.2,56.3$; FT-IR (KBr)
$3060,2945,1640(\mathrm{C}=0), 1485,1362,1030 \mathrm{~cm}^{-1} ; \mathrm{Ms} m / z$ (\%) $252\left(\mathrm{M}^{+}, 100\right), 251(83), 222(25), 150(39), 107(18)$.

5-Hydroxy-4'-chloroflavone (5h). M.p. $190-191{ }^{\circ} \mathrm{C} ;{ }^{1} \mathrm{H}$ $\mathrm{NMR}\left(300 \mathrm{MHz}, \mathrm{CDCl}_{3}\right) \delta 12.50(\mathrm{~s}, 1 \mathrm{H}), 7.84$ (d, $J=8.8$ $\mathrm{Hz}, 2 \mathrm{H}), 7.54\left(\mathrm{dd}, J_{1}=8.4 \mathrm{~Hz}, J_{2}=8.3 \mathrm{~Hz}, 1 \mathrm{H}\right), 7.50(\mathrm{~d}, J=$ $8.8 \mathrm{~Hz}, 2 \mathrm{H}), 6.99\left(\mathrm{dd}, J_{1}=8.4 \mathrm{~Hz}, J_{2}=0.8 \mathrm{~Hz}, 1 \mathrm{H}\right), 6.82$ $\left(\mathrm{dd}, J_{1}=8.3 \mathrm{~Hz}, J_{2}=0.8 \mathrm{~Hz}, 1 \mathrm{H}\right), 6.70(\mathrm{~s}, 1 \mathrm{H}) ;{ }^{13} \mathrm{C} \mathrm{NMR}$ $\left(75 \mathrm{MHz}, \mathrm{CDCl}_{3}\right) \delta 183.8,163.7,161.2,156.7,138.8,135.9$, $130.0,129.9,128.1,112.0,111.2,107.4,106.5$; FT-IR (KBr) $3434(\mathrm{OH}), 3075,1659(\mathrm{C}=\mathrm{O}), 1621,1265,1113,830,746$ $\mathrm{cm}^{-1} ; \mathrm{Ms} m / 2(\%) 274\left(\mathrm{M}^{+}+2,34\right), 272\left(\mathrm{M}^{+}, 100\right), 244(14)$, $136(55), 108(55), 77(12)$.

Acknowledgment. This research was financially supported by a grant (R06-2004-004-01001-0) from the Korea Science and Engineering Foundation.

\section{References}

I. Harborne, J. B.; Williams, C. A. Nat. Prod. Rep. $2001,18,310$.

2. (a) Tsuchiya, Y; Shimizu, M.; Hiyama, Y.; Jtoh, K.; Hashimoto, Y.; Nakayama, M.; Horie, T; Morita, N. Chem. Phorm. Bull. 1985, 33, 3881. (b) Middleton, E.; Kandaswami, C. Food Technol. 1994, 48, 1 15. (c) Bors, W.; Michel, C.; Stettmaier, K. BioFoctors 1997, 6, 399. (d) Nijveldt, R. J.; Nood, E.; Hoorm, D.; Boelens, P. G.; Norten, K.; Leeuwen, P, Am. J. Clin. Nitr: 2001, 74, 418.

3. Bohm, B. A. Introduction to Flanonoids; Harwood Academic Publishers: Amsterdam. Netherlands. 1998; p 243.

4. (a) Wheeler, T. S. Org. Synth. Coll. Vol. 4, 1963, 478. (b) Nishinaga. A.: Ando. H.; Maruyama. K.; Mashino. T. Symthesis 1992, 839. (c) Ares, J. J.; Outl, P. E.; Kakodkar, S. V.; Buss, R. C.; Geiger, J. C. J. Org. Chem. 1993, 58, 7903. (d) Zembower, D. E.; Zhang, H. ibid. 1998, 63, 9300 .

5. (a) Banerij, A.; Goomer, N. C. Syththesis 1980, 874. (b) Saxena, S.; Makrandi, J. K.; Grover, S. K. ibid. 1985, 697. (c) Cushman, M.; Nagarathnam, D. Tetrahedron Lett. 1990, 3/, 6497.

6. (a) Nagarathnam, D.; Cushman, M. J. Org. Chem. 1991, 56, 4884. (b) Nagarathnam, D.; Cushman, M. Tetrahedron 1991, 47, 5071. (c) Menichincheri, M.; Ballinari, D.; Bargiotti, A.; Bonomini, L.; Ceccarelli, W.; D'Alessio, R.; Fretta, A.; Moll, J.; Polucci, P.; Soncini, C.; Tibolla, M.; Trosset, J. Y.; Vanotti, E. J. Med. Chem. 2004. 47.6466

7. (a) Kasahara, A.; Izumi, T.; Ooshima, M. Bull. Chem. Soc. Jpn. 1974, 47, 2526. (b) Ali, S. M.; Iqbal, J.; Ilyas, M. Chem, and Ind. 1985, 276. (c) Makrandi, J. K.; Seema ibid. 1989, 607. (d) Hans, N.; Grover, S. K. Symh. Comm. 1993, 23, 1021. (e) Litkei, G.; Gulacsi, K.; Antus, S.; Blasko, G. Liebigs Amm. 1995, 1711. (f) Miyake, H.; Takizawa, E.; Sasaki, M. Bull. Chen. Soc. Jpn. 2003. 76,835 .

8. (a) Hercouet, A.; Corre, M. L. Synthesis 1982, 597. (b) Floch, Y. L.; Lefeuvre, M. Tetrahedron Lett. 1986, 27, 2751.

9. Staunton, J. In Comprehensive Organic Chenistry; Barton, D.; Ollis, W. D., Eds.; Pegamon Press: Oxford, U. K., 1979; Vol. 4, p 679.

10. Blasko, G; Xun, L.; Cordell, G. A.J. Nat. Prod, 1988, 51, 60.

11. Lee, J. I.; Son, H. S.; Park, H. Bull. Korean Chem. Soc. 2004, 25, 1945. 